\title{
A FORMAÇÃO PROFISSIONAL DO ENGENHEIRO: UM ENFOQUE NAS METODOLOGIAS ATIVAS DE APRENDIZAGEM EM UNIVERSIDADE FEDERAL
}

\author{
Ricardo Luiz Perez Teixeira*, Cynthia Helena Soares Bouças Teixeira*, Max Leandro \\ de Araújo Brito** \\ *Universidade Federal de Itajubá; **Universidade Federal do Rio Grande do Norte \\ DOI: 10.15628/rbept.2018.7330
}

Artigo submetido em maio/2018 e aceito em out/2018

\begin{abstract}
RESUMO
O presente estudo tem por objetivo discutir a formação profissional do engenheiro a partir da experiência em um curso de engenharia em uma Universidade Federal brasileira. A metodologia utilizada foi quali-quantitativa, consistindo na análise da utilização de Metodologias Ativas em duas disciplinas do curso de Engenharia de Materiais da Universidade Federal de Itajubá, Campus de Itabira. Os resultados indicaram que os discentes são favoráveis e demandam a utilização dessas metodologias. O estudo conclui que a experiência no curso de engenharia da Universidade é relevante para a formação profissional do engenheiro, porém as instituições brasileiras ainda não se adequaram totalmente às novas demandas das políticas educacionais, o que exige uma readequação nos currículos de engenharia e seus Projetos Políticos Pedagógicos.
\end{abstract}

Palavras-Chave: Educação profissional. Ensino de engenharia. Aprendizagem baseada em problemas.

\section{VOCATIONAL TRAINING OF THE ENGINEER: AN APPROACH TO ACTIVE LEARNING METHODOLOGIES IN FEDERAL UNIVERSITY}

\begin{abstract}
O The present study aims to discuss the professional training of the engineer from the experience in an engineering course at a Brazilian Federal University. The methodology used was qualitative and quantitative, consisting of the analysis of the use of Active Methodologies in two subjects of the Materials Engineering course of the Federal University of Itajubá, Itabira Campus. The results indicated that the students are favorable and require the use of these methodologies. The study concludes that the experience in the engineering course of the University is relevant to the professional training of the engineer, but the Brazilian institutions have not yet fully adapted to the new demands of the educational policies, which requires a re-adaptation in the engineering curricula and its Political Projects Pedagogical.
\end{abstract}

Keywords: Professional education Engineering education. Problem-based learning. 


\section{INTRODUÇÃO}

Pesquisadores da área de educação vêm buscando as metodologias ativas para um aprendizado mais participativo dos discentes numa nova postura docente na colaboração do aprendizado (SANTOS; SHITSUKA; TEIXEIRA, 2014; REZENDE JÚNIOR et al., 2013). A reflexão e a intervenção nos currículos de engenharia, aliando habilidades técnicas que devem ser desenvolvidas no profissional e as melhores formas de aprendizagem ativa pelo discente, tem sido o principal foco em sua implantação (BOGHI et al., 2016; MORAN, 2015; TEIXEIRA, 2014; REZENDE JÚNIOR et al., 2013).

No Projeto de Implantação de Metodologias Ativas na Universidade Federal de Itajubá Campus de Itabira, avaliou-se o uso de metodologias ativas no curso de Engenharia de Materiais da UNIFEI Campus de Itabira (EMT), concentrando-se no estudo e análise da "Aprendizagem Baseada em Problemas" (do inglês: Problem-Based Learning - PBL).

O PBL é uma metodologia de ensino-aprendizagem na qual situaçõesproblema fomentam a aprendizagem e consolidação de conceitos, teorias e 0 desenvolvimento de habilidades e atitudes no contexto de sala de aula. Para um melhor aproveitamento da metodologia ativa em engenharia e cursos técnicos, as atividades devem ter conexão com as habilidades de demandadas na profissão e ao mesmo tempo permitir a descoberta e a análise crítica por parte do discente, como relatado por Teixeira e Teixeira (2017) e Rezende Júnior et al (2013). Trata de resolução de possíveis problemas reais em classe, demandando pesquisa em literatura de referência para a área e outras construídas no contexto do problema atual e presente (GORGES; PASSOS; WOLLINGER, 2018; BERBEL; GAMBOA, 2012; BERBEL, 2011).

Teixeira (2014) e Rezende Júnior et al (2013) ressaltam como inapropriada adequar a aprendizagem tradicional ao novo profissional proativo e conectado às novas realidades virtuais, transcendendo, assim, a antiga metodologia de transmissão/recepção de conhecimento fixo. Tem-se, então, uma demanda por mudanças na metodologia de ensino e nas estratégias de docência, seja por modelos educacionais alternativos aos convencionais ou por modelos mais adequados baseados em metodologias ativas (REZENDE JÚNIOR et al., 2013; STROHSCHOEN. et al, 2018).

Tendo em vista o contexto apresentado, o presente artigo tem por objetivo discutir a formação profissional do engenheiro a partir da experiência em um curso de engenharia em uma Universidade Federal brasileira. Desse modo, realiza uma breve revisão sobre a formação profissional do engenheiro e as metodologias ativas no ensino de engenharia, seguida da metodologia, resultados e discussões e, por fim, as considerações finais do estudo. 


\section{A FORMAÇÃO PROFISSIONAL DO ENGENHEIRO E AS METODOLOGIAS ATIVAS}

Com o desenvolvimento econômico registrado no Brasil nos últimos 20 anos e a cobrança do mercado por profissionais qualificados em nível de formação superior foram desenvolvidas ações estatais voltadas às exigências do mercado e às cobranças da sociedade por mais desenvolvimento do ensino superior no país. Deste modo ocorreu criação e ampliação das políticas públicas de garantia de acesso e permanência no ensino superior, com preocupações em relação à melhoria do ensino, com o fortalecimento dos exames nacionais de avaliação dos estudantes (CARMO et al, 2015).

A democratização do ensino superior ocorreu em várias frentes, dentre eles As principais iniciativas de facilitação do acesso aos cursos superiores foram o Programa Universidade para Todos (ProUni) e o Programa de Apoio a Planos de Reestruturação e Expansão das Universidades Federais (Reuni), e o Fundo de Financiamento Estudantil (Fies) (CARMO et al, 2014); a reserva de vagas nas universidades públicas para estudantes oriundos de escolas públicas, além das cotas raciais e étnicas, promoveram o acesso ao ensino superior à população historicamente menos favorecida (CARMO et al, 2015).

Essa democratização do ensino superior também alcançou os cursos de engenharia, que no Brasil tem tradição de formação de engenheiros em uma formação longa, com perfil inicial generalista e formação final técnica especializada, orientada para o mercado de trabalho regional. $O$ ensino de engenharia foi ajudado por uma política de substituição das importações que buscou criar uma indústria nacional mais abrangente a partir da década de 70 , que aumentou gradativamente a demanda por engenheiros com formação mais científica e maior conhecimento técnico. Com o tempo ocorreu um inchaço dos currículos de engenharia com disciplinas de ciências básicas sem uma maior integração com as disciplinas profissionais. A partir de então pensa-se na necessidade de tornar o engenheiro empreendedor, com base científica suficiente para acompanhar mudanças tecnológicas (ROHAN et al, 2016).

A utilização de metodologias de ensino tradicionais, com absorção do conteúdo por meio de referenciais teóricos na lousa, transcritos para o caderno dos estudantes, sem a interação dos mesmos, foi amplamente desenvolvida no ensino de engenharia no Brasil. Porém, a busca por engenheiros capazes de atender às novas necessidades do mercado e que entendam a realidade enfrentada pelas empresas, fez aumentar a procura por metodologias ativas no ensino de engenharia no Brasil (PONCIANO; GOMES; MORAIS, 2017).

A metodologia da Aprendizagem Baseada em Problemas (PBL - em inglês) é uma das mais utilizadas nas engenharias. Nela o professor passa a mediar a aprendizagem do estudante e tem o "problema" como elemento 
motivador do estudo e integrador do conhecimento. A abordagem procura desenvolver as múltiplas habilidades do aluno, por meio do equilíbrio entre a teoria e prática, aumentando a motivação do estudante com casos práticos (PONCIANO; GOMES; MORAIS, 2017).

\section{METODOLOGIA}

A metodologia utilizada foi quali-quantitativa, consistindo na análise da utilização de Metodologias Ativas em duas disciplinas do curso de Engenharia de Materiais da Universidade Federal de Itajubá, Campus de Itabira. Apesar de a metodologia de ensino das disciplinas não constar formalmente no Plano Pedagógico atual do curso de Engenharia de Materiais da UNIFEI Campus de Itabira (UNIFEI, 2018), a UNIFEI fomenta a utilização de metodologias ativas por parte docente, desde que esteja previsto no plano de ensino docente, que não haja perda na aprendizagem discente e que esteja dentro do currículo da formação do profissional.

A seguir, serão detalhadas as experiências em algumas disciplinas do Curso de Engenharia de Materiais da UNIFEI Campus de Itabira.

A disciplina de Ciências dos Materiais é oferecida pelo docente Prof. Dr. Ricardo Luiz e utiliza parcialmente a Aprendizagem Baseada em Problemas (PBL), onde os alunos são fomentados a associar o conhecimento da literatura de referência com o aprendizado prático e laboratorial.

A disciplina é dividida em quatro módulos: (1) Introdução à ciência e à engenharia dos materiais (base teórica); (2) Ligações químicas e estruturas cristalinas; (3) Propriedades dos diversos tipos de materiais; (4) Caracterização química e cristalográfica dos diversos tipos de materiais. A disciplina foi dividida também em duas fases: a fase 1 corresponde a apresentação dos resultados sem consistência estatística dos módulos no meio do período da disciplina e a fase 2 são os resultados da fase 1 tratados estatisticamente e com uma maior representatividade científica em confiança dos resultados.

Destacam-se como resultados encontrados pelos discentes na solução de problemas propostos pelo docente: a identificação de dois materiais metálicos diferentes em termos de propriedades mecânicas, composição química e tipo microestrutura com uma confiança mínima de 95\% dos resultados.

Como exemplo de resultados promissores na oferta da disciplina de Ciências dos Materiais, os alunos apresentaram em forma de relatório técnico e seminário os resultados e considerações relativos à identificação dos dois materiais metálicos, bem como o grau de confiança quanto a afirmação das conclusões quanto aos tipos de materiais metálicos identificados. 
A disciplina de Processos de Manufatura tem por objetivo apresentar os diversos tipos de conformação plástica de metais para o EMT, além do grupo de estudantes do curso de Engenharia Mecânica.

Destacam-se como resultados encontrados pelos discentes na solução de problemas propostos pelo docente: o comparativo de dois processos diferentes de conformação plástica de um mesmo material metálico para uma destinação única e comum. Os discentes avaliaram a conformação dos metais em termos de propriedades mecânicas, microestrutura obtida após a conformação e a velocidade de conformação com uma confiança mínima de 95\% dos resultados.

A disciplina é dividida em três módulos: (1) Introdução à conformação plástica de metais; (2) Conformação Plástica Mecânica e (3) Conformação Plástica Metalúrgica. A experiência do docente Prof. Dr. Ricardo Luiz contribuiu para a escolha da metodologia ativa PBL utilizada integralmente nessa disciplina. A disciplina foi dividida também em duas fases: a fase 1 corresponde a apresentação dos resultados sem consistência estatística nos módulos no meio do período da disciplina e a fase 2 são os resultados da fase 1 tratados estatisticamente e com uma maior representatividade científica em confiança dos resultados.

$\mathrm{Na}$ Fase 1, tem-se a contextualização dos problemas. Na turma do segundo semestre de 2016, o primeiro problema consistiu em conhecer, discutir e sedimentar o aprendizado em Engenharia e Ciência dos Materiais. O segundo problema, consistiu em uma grande atividade de contatar uma empresa que produz ou processa o material metálico conforme proposto em classe.

O docente facilitador fomentou a atividade discente em PBL dispondoos em ilhas. Nas ilhas discentes, elege-se entre si líderes, trabalhadores e "escrivães" para responder importantes perguntas no campo vasto de Engenharia e Ciência dos Materiais e de Processos de Manufatura antes do contato com a empresa produtora ou conformadora plástica do metal: (a) "O que se quer conhecer?", (b) "Como agir na busca do conhecimento?"; (c) "Como utilizar e conectar os conhecimentos adquiridos entre si?"; (d) "Como registrar o que se conhece?"; (e) "Como avaliar o registro obtido?"; e (f) "Como apresentar os resultados obtidos estatisticamente no Seminário na Fase 2?".

Consiste na solução de três problemas PBL propostos. Cada ilha discente no PBL apresentou a "solução" para o problema proposto ao discutir o tipo de material, suas características, propriedades, processos de conformação (para disciplina Processos de Manufatura), tamanho amostral, população amostral, avaliação da confiança da média amostral e seu desvio padrão.

O resultado mais expressivo foi a elaboração de um relatório técnico descritivo com um grau de confiança de $95 \%$ dos resultados e conclusões obtidos a partir da experiência prática discente. 


\section{RESULTADOS E DISCUSSÕES}

Baseando-se no trabalho de REZENDE JÚNIOR et al (2013), tem-se no Gráfico 1 a parte da avaliação quantitativa de sete critérios coletados por meio da Avaliação do Processo Educacional (APE) nas duas Fases da disciplina Ciência dos Materiais em conjunto com os de Processo de Manufatura. Pelos resultados, tem-se uma avaliação geral bem positiva com média global 4,2; sendo que o 1,0 representa o conceito (I) Insuficiente, 2,0 representa o conceito (R) Regular, 3,0 representa o conceito (B) Bom, 4,0 representa o conceito (O) Ótimo e 5,0 representa o conceito (E) Excelente.

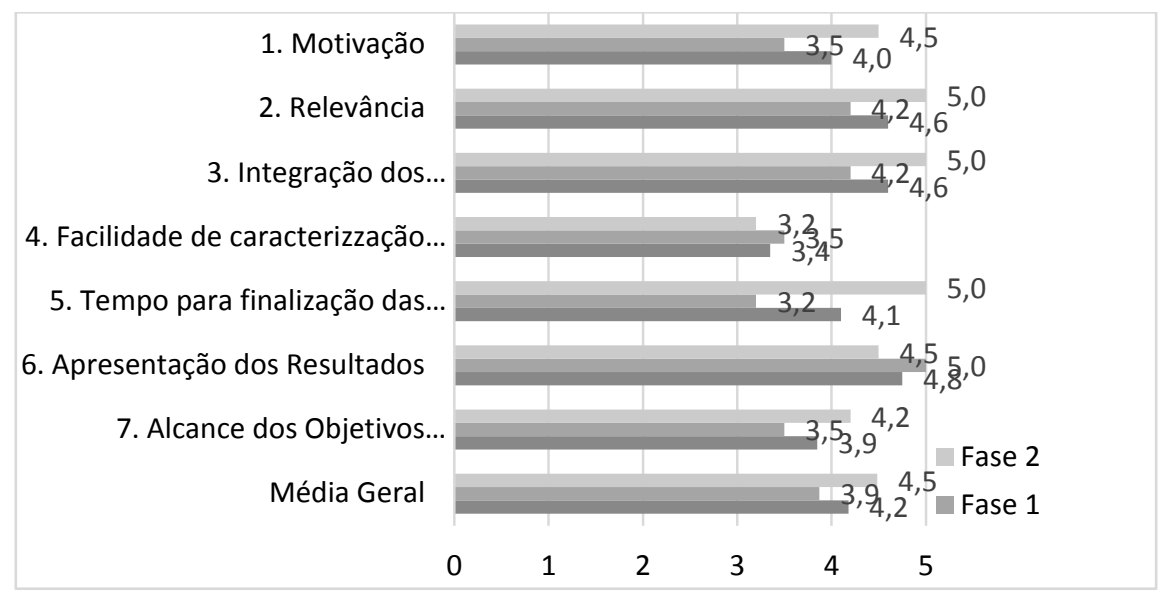

Figura 1: Consolidação de parte da Avaliação do Processo Educacional (APE)

Observa-se ainda no Gráfico 1 uma avaliação boa para o "Tempo de finalização das atividades", acredita-se que o resultado não foi melhor devido ao contato inédito discente na Fase $1 \mathrm{com}$ uma nova metodologia PBL (média 4,1, considerado (O) Ótimo), o que demandou mais tempo de aprendizagem e entendimento inicial discente (REZENDE JÚNIOR et al., 2013). Entretanto, o critério "Relevância" junto a "Integração dos resultados" do método foram os critérios de destaque na avaliação (média superior a 4,5, considerado (O) Ótimo), o que indica que o método PBL está adequado para a disciplina. Seguem alguns comentários de discentes da turma do segundo semestre de 2016, com respeito à avaliação qualitativa do método PBL utilizado, parte integrante das disciplinas de EMT.

Quando perguntados sobre a metodologia ativa utilizada na disciplina os alunos responderam apontando para o desafio que é esta metodologia e a importância de se ter a prática já no contexto da sala de aula, conforme podese observar no Quadro 1. 
Quadro 1: Opinião sobre a metodologia

\begin{tabular}{|c|l|c|}
\hline \multicolumn{1}{|c|}{ Categoria } & \multicolumn{1}{|c|}{ Comentário } & Sujeito \\
\hline \multirow{2}{*}{ Desafiadora } & $\begin{array}{l}\text { O método de ABP me deu muito trabalho, imagina } \\
\text { para o professor }\end{array}$ & B \\
\cline { 2 - 3 } $\begin{array}{l}\text { A pessoa não deve ter vergonha de apresentar um } \\
\text { resultado quando tem todas as informações com } \\
\text { confiança, adorei } \\
\text { prática para a a }\end{array}$ & $\begin{array}{l}\text { Foi legal refletir sobre a representatividade dos } \\
\text { resultados }\end{array}$ & A \\
\cline { 2 - 3 } & $\begin{array}{l}\text { O método é bem ir na prática, ver, pesquisar, } \\
\text { refletir, repetir, fazer considerações é um método } \\
\text { científico, não? }\end{array}$ & C \\
\hline
\end{tabular}

Em relação ao aprendizado quando utilizada a metodologia ativa, as respostas dos discentes informam que a metodologia favorece a promoção de novas descobertas, além de fidelizar o aluno, tornando-o mais interessado nesta forma dinâmica de ensino e aprendizagem, conforme apresentado no Quadro 3.

Quadro 2: Opinião sobre o aprendizado

\begin{tabular}{|l|l|c|}
\hline \multicolumn{1}{|c|}{ Categoria } & \multicolumn{1}{|c|}{ Comentário } & Sujeito \\
\hline $\begin{array}{l}\text { Promove novas } \\
\text { descobertas }\end{array}$ & $\begin{array}{l}\text { Essa foi a oportunidade de ir ao laboratório de } \\
\text { metalografia pela primeira vez }\end{array}$ & A \\
\hline \multirow{2}{*}{ Fideliza o aluno } & Considero a aprendizagem muito atraente & B \\
\cline { 2 - 3 } & $\begin{array}{l}\text { Ótimo, por que não adotar em todas as disciplinas } \\
\text { este método? }\end{array}$ & D \\
\hline
\end{tabular}

\section{CONSIDERAÇÕES FINAIS}

O estudo conclui que a experiência no curso de engenharia da Universidade é relevante para a formação profissional do engenheiro, porém as instituições brasileiras ainda não se adequaram totalmente às novas demandas das políticas educacionais, o que exige uma readequação nos currículos de engenharia e seus Projetos Políticos Pedagógicos.

Os novos profissionais proativos em engenharia e técnicos demandam novas metodologias ativas, como a PBL, porém para tal se faz necessária uma readequação nos currículos de engenharia e seus PPCs, bem como de uma melhor receptividade tanto de docentes como de discentes. A UNIFEI 
Campus de Itabira esforça-se para suprir essa demanda através de treinamento de seus docentes em metodologias ativas e já há boas experiências de utilização de metodologias ativas, como relatado nesse artigo.

\section{REFERÊNCIAS}

BERBEL, N. A. N. As metodologias ativas e a promoção da autonomia de estudantes. Semina: Ciências Sociais e Humanas, v. 32, n. 1, p. 25-40, 2011.

BERBEL, N. A. N.; GAMBOA, S. A. S. A metodologia da problematização com o Arco de Maguerez: uma perspectiva teórica e epistemológica. Revista Filosofia e Educação on-line, Campinas, v. 3, n. 2, p. 264-287, 2012.

BOGHI, C. et al. Metodologia ativa: um estudo de pesquisa-ação na disciplina de termodinâmica em um curso de engenharia. Tecnologia Educacional, v. 214, p. 70-82, 2016.

CARMO, E. F. et al. Políticas públicas de democratização do acesso ao ensino superior e estrutura básica de formação no ensino médio regular. Rev. bras. Estud. pedagog., Brasília, v. 95, n. 240, p. 304-327, 2014.

CARMO, E. F., et al. Discutindo as políticas públicas de acesso ao ensino superior. Revista Educação, v.10, n.1, 17-28, 2015.

GORGES, S.; PASSOS, A. P. P.; WOLLINGER, H. Competências do administrador: um estudo com acadêmicos do curso de administração no contexto da aprendizagem ativa. Research, Society and Development, v. 7, n. 1, p. 1-27, e471120, 2018.

MORAN, J. M. Mudando a educação com metodologias ativas. In: Coleção Mídias Contemporâneas. Convergências Midiáticas, Educação e Cidadania: aproximações jovens. Vol. II. Carlos Alberto de Souza e Ofelia Elisa Torres Morales (orgs.). PG: Foca Foto-PROEX/UEPG, 2015.

PONCIANO, T. M.; GOMES, F. C. V.; MORAIS, I. C. Metodologia ativa na engenharia: verificação da ABP em uma disciplina de engenharia de produção e um modelo passo a passo. Princípia, n. 34, p. 32-39, 2017.

REZENDE JÚNIOR, R. A. et al. Aplicabilidade de metodologias ativas em cursos de graduação em engenharia. In: CONGRESSO BRASILEIRO DE EDUCAÇÃO EM ENGENHARIA, 41, 2013, Gramado. Anais... Gramado: Cobenge, 2013.

ROHAN, U. et al. A formação do engenheiro civil inovador brasileiro frente aos desafios da tecnologia, do mercado, da inovação e da sustentabilidade. In: CONGRESSO NACIONAL DE EXCELÊNCIA EM GESTÃO, 12., 2016, Rio de Janeiro. Anais... Rio de Janeiro: CNEG, 2016.

SANTOS, J. B.; SHITSUKA, R.; TEIXEIRA, R. L. P. A construção do raciocínio lógico-dedutivo para alunos dos cursos de engenharia metalúrgica. 
Maestria (Sete Lagoas), v. 12, n. 1, p. 99-117, 2014.

STROHSCHOEN, A. A. G. et al. Mestrados para a Formação de Docentes: um locus de (re) construção e de aprendizagem. Research, Society and Development, v. 7, n. 8, p. 01-17, e378324, 2018.

TEIXEIRA, R. L. P. A escola cidadã: a avaliação formativa como formativa como ferramenta transformadora para uma escola cidadã. Maestria (S. Lagoas), v. 11, n. 1, p. 135-146, 2014.

TEIXEIRA, R. L. P. TEIXEIRA, C. H. S. B. Um modelo de Construção do conhecimento através da prática investigativa de corrosão. Research, Society and Development, v. 4, n. 1, p. 38-44, 2017.

UNIFEI. PPC do curso de Engenharia de Materiais da UNIFEI, Campus de Itabira. Itabira: DAI, 2018. 